\section{Análisis de los niveles de automatización de los procesos industriales}

\author{
Analysis of the levels of automation of industrial \\ processes
}

\section{elSSN: 2600-5743}

revistacentrosur@gmail.com Recepción: 4 octubre 2019 Aprobación 12 noviembre 2019 Pag $16-23$

Atribución/ReconocimientoNoComercial-Compartirlgual 4.0 Licencia Pública Internacional CC BY-NC-SA 4.0 https://creativecommons.org/lice nses/by-nc-sa/4.0/legalcode.es

\author{
Gabriel Pico Saltos ${ }^{1}$ \\ Josselyn Maricel Murillo Villaprado ${ }^{2}$ \\ Fernando Vladimir Pincay de la Rosa ${ }^{3}$ \\ Faustino Raúl Pincay Villacréses ${ }^{4}$
}

\section{Resumen}

El presente proyecto de investigación está enfocado en el análisis de los niveles de automatización de los procesos industriales de la empresa Unidad de Negocios Termopichincha Quevedo II CELEC EP con el fin de identificar los problemas $y$ oportunidades de mejora que permitan el aumento de la eficiencia y el incremento de la productividad. Cada uno de los métodos utilizado en la investigación como el método inductivo, de observación y analítico han sido de vital importancia para llegar a los resultados y conclusiones finales; donde se ha determinado por medio de una entrevista realizada al jefe encargado de la empresa, Ing. Gabriel Robinson que la empresa mantiene un nivel 5 de automatización correspondiente a la pirámide del mismo, debido a que la mayoría de sus procesos son automatizados a excepción del proceso de recepción de combustible y agua cruda el cual requiere mejoras en automatización. Por ende, se ha propuesto la utilización de una boya industrial para minimizar pérdidas de agua $y$ tiempos innecesarios de monitoreo al momento

1 Máster Universitario en Energías Renovables en Sistemas Eléctricos. Universidad Técnica Estatal de Quevedo. Quevedo - Ecuador. Email: Ipico@uteq.edu.ec. ORCID: https://orcid.org/0000-0002- 9668 - 9660

Google académico:

https://scholar.google.es/citations?hl=es\&user=svOBKIcA AAAJ

2 Quinto año de Ingeniería Industrial. Universidad Técnica Estatal de Quevedo. Quevedo - Ecuador. Orcid: https://orcid.org/0000-0002-0299-0728. Email: murillojosselin96@gmail.com Google scholar:

https://scholar.google.es/citations?view_op=new_articles \&hl=es\&imq=JOSSELIN+MARICEL+MÜRILLO+VILLAPR ADO\#

3 Quinto año de Ingeniería Industrial. Universidad Técnica Estatal de Quevedo. Quevedo - Ecuador. Orcid: https://orcid.org/0000-0001-5341-7756. Email: fernando.pincay2015@uteq.edu.ec

Google scholar:

https://scholar.google.es/citations?view_op=new_articles \&hl=es\&imq=Fernando+Vladimir+Pincay+de+la+Rosa\# 4 Quinto año de Ingeniería Industrial. Universidad Técnica Estatal de Quevedo Quevedo - Ecuador. Orcid: https://orcid.org/0000-0002-9784-5485. Email: faustino.pincay2015@uteq.edu.ec Google scholar: https://scholar.google.es/citations?view_op=new_articles \&hl=es\&imq=FAUSTINO+RAUL+PINCAY+VILLACRESE S\# 
de llenado dentro del proceso de recepción de agua cruda. El resultado del estudio es la descripción y evaluación de las alternativas de acuerdo a los problemas, necesidades y posibilidades de implementación teniendo en cuenta la infraestructura con que cuenta la Empresa y a la proyección de la misma.

Palabras clave: infraestructura, evaluación, interpretación

\section{ABSTRACT}

The present research project is focused on the analysis of the levels of automation of the industrial processes of the company Business Unit Termopichincha Quevedo II CELEC EP in order to identify the problems and opportunities of improvement that will allow the increase of the efficiency and the improvement increase in productivity. Each of the methods used in research as the inductive, observational and analytical method has been of vital importance in reaching the final results and conclusions; from where it was determined by means of an interview conducted by the head of the company, Ing. Gabriel Robinson that the company maintains a level 5 of automation corresponding to the pyramid of the same, due to the fact that most of its processes are automated at

exception of the process of reception of fuel and raw water which requires better automation. Finally, the use of an industrial boy has been proposed to minimize water losses and unnecessary monitoring time at the time of drawing inside the raw water reception process. The result of the study is the description and evaluation of the alternatives to meet the problems, needs and possibilities of implementation, depending on the infrastructure with which the Company and the project of same.

Keywords: infrastructure, evaluation, interpretation

\section{Introducción}

Hoy en día, las empresas deben enfrentarse a un mundo globalizado en el cual, el cliente es el elemento más importante, puesto que es el que determina la demanda de los productos y/o servicios ofrecidos por estas; razón por la cual se debe responder de manera oportuna y eficiente a sus expectativas y necesidades (Sabogal Vanegas, 2012).

Actualmente, es importante mantener un alto nivel de calidad en los productos y servicios, lo cual puede ser el resultado de la calidad en los procesos productivos y administrativos que se realizan dentro de la organización. Uno de los factores más importantes para poder ser competitivas es la productividad que está relacionada con los métodos de trabajo, los procesos eficientes, la mejora continua y el uso o implementación de la tecnología. 
El presente proyecto tiene como objetivo el análisis de los niveles de automatización de los procesos industriales de la empresa Unidad de Negocios Termopichincha Quevedo II CELEC EP, proponiendo alternativas de solución basadas en la tecnología de automatización industrial con el fin de mejorar la eficiencia del proceso de distribución interna, el cual inicia con la recepción de combustible y finaliza con la distribución de la energía generada.

Para lograr estos objetivos se realizaron visitas de campo a la planta de generación eléctrica la cual tenía un sistema de recepción de agua cruda no automatizada. Con la ayuda de los datos recolectados en el campo, entrevista realizada al jefe encargado, se procedió a receptar datos de funcionamiento de cada uno de los procesos que mantiene la empresa para poder llegar a la respectiva solución. En este proyecto de investigación se analizará cada uno de los niveles de automatización que mantiene la empresa, lo cual disminuirá los tiempos elevados de trabajo. Además, con la incorporación de la tecnología de automatización se disminuyen los errores en los diferentes procesos, se presenta una mayor agilidad en la entrega de los pedidos y se reduce los inventarios en procesos.

Un proceso industrial es el conjunto de etapas que hacen posible la transformación de la materia prima e insumos en productos, subproductos, residuos y desechos; usando racionalmente la energía, y teniendo en cuenta en cada etapa las condiciones de operación que hagan posibles procesos eficientes. Las etapas son actividades unitarias que pueden ser operaciones unitarias o procesos unitarios, aunque entre algunas de ellas la diferencia es muy sutil y en otras se complementan (Loayza Pérez \& Silva Meza, 2013).

Los procesos químicos industriales sostenibles o procesos industriales sostenibles, son procesos también constituidos por etapas que son actividades unitarias, pero que potencian el aprovechamiento de los materiales y la energía para la producción de bienes (o productos útiles) y minimizan o eliminan la presencia de residuos y desechos -o males- (ya que dependiendo del tipo de residuo, estos pueden contribuir a la contaminación ambiental y a sus efectos) (teacherke, 2010).

\section{Tipos de procesos industriales}

El aprovechamiento de los recursos naturales, el trabajo de calidad y la flexibilidad ante diversos cambios son algunos de los factores que permiten establecer ciertas diferencias entre procesos de este tipo, los cuales se clasifican básicamente en 4 categorías: (Blanca, 2008)

\section{Procesos por lotes}

Son aquellos que aceleran el volumen de producción con la ayuda de plantillas o modelos. En muchos sectores de la economía, este tipo de procesos han suplido a otros de carácter artesanal. El volumen de los artículos producidos lo determinan las necesidades de cada empresa y el compromiso con sus clientes (School).

\section{Procesos por flujo continuo}


En este caso, las empresas realizan cientos de miles de productos idénticos. Lo que les diferencia de otros es que la línea de producción nunca cesa; es decir, está en funcionamiento las 24 horas del día y los 7 días de la semana. El objetivo de este modelo es doble: por un lado, maximizar los niveles de producción de la empresa; por otro, reducir los costes generados a raíz de detener e iniciar una vez tras otra el proceso industrial (MCR, 2016).

\section{Procesos por trabajo}

La modalidad consiste en centrar todos los esfuerzos productivos en la elaboración de un producto cada vez. Por ejemplo, en función de un pedido específico o de una temporada concreta. El resultado es distinto en cada ocasión y el reto consiste en mantener la identidad de la línea de producción y la calidad (Siemens).

\section{Producción en masa}

Es uno de los modelos más empleados en la industria. Al igual que la producción por flujo continuo, contempla la elaboración de cientos de miles de artículos en una misma serie, con la diferencia de que la producción no es permanente; hay unos plazos de inicio y de fin de ciclo. Al tratarse de un alto volumen de artículos, este tipo de procesos supone el uso de sistemas de alta tecnología (Fernández Sánchez)

\section{Mercado eléctrico ecuatoriano}

En los últimos años en el mercado eléctrico ecuatoriano se ha registrado un crecimiento de la demanda mayor al crecimiento de la oferta, parcialmente este déficit ha sido cubierto, a través de la generación térmica y la importación de energía, que trae como consecuencia una afección directa al precio para el consumidor final (Robles, 2010).

Las administraciones en general de las distribuidoras han sido poco acertadas, sin una planificación previa a largo plazo, fijación de tarifas irreales causando pérdidas lo que ha llevado a una crisis del sector eléctrico en el campo financiero y consecuentemente en la calidad del servicio.

Las empresas de distribución juegan un papel importante para hacer frente a la crisis que vive el sector eléctrico ecuatoriano, tanto problemas financieros como otras condiciones negativas que afectan la estabilidad de este importante sector. (Taipe Flores, 2011)

\section{Materiales y métodos}

La Unidad de Negocios Termopichincha Quevedo II CELEC EP se encuentra ubicada en el $\mathrm{km} 1 \frac{1}{2}$ vía El Empalme, ciudadela la Virginia diagonal a la Escuela de Clases de la Policía Nacional del Club de Tropas. La investigación que se está realizando es de tipo diagnostica porque se basa en un análisis de los niveles de automatización, interpretando cada uno de los posibles problemas que se encuentran presente en la Empresa. Los métodos que se van a llevar a cabo durante todo el proceso de investigación son los siguientes: Se va a emplear este método debido a que se 
realizara entrevistas al jefe encargado de la Central, supervisores de operación y personal que labora dentro de la misma. La ejecución de este método se la empleará para el levantamiento de información a través de visitas a la empresa con el fin de obtener conocimientos acerca del funcionamiento de todos los procesos realizados en la central. Además de adquirir datos con respecto a los niveles de automatización y los procesos automatizados en la Unidad de Negocio Termopichincha Quevedo II CELEC EP. Se efectuará el respectivo análisis en cada una de las áreas de la Empresa mediante la cual se determinará el nivel de automatización actual con el que cuenta la Central, además nos ayudará para el desarrollo de las debidas conclusiones. El presente proyecto tendrá un diseño no experimental porque está contemplado en realizar un análisis detallado de los niveles de automatización, por lo tanto, no se desarrollará ningún tipo de experimento. En el presente proyecto de investigación se realizará las respectivas entrevistas al Jefe de la Central encargado el Ing. Gabriel Robinson, dentro de los cuales se darán a conocer el funcionamiento, procesos dentro de la misma; además se desarrollará observación directa para determinar e identificar los posibles problemas que mantiene la Central con respecto a los niveles de automatización actual. Esto será de ayuda para la respectiva ejecución de los resultados. Para el tratamiento de datos obtenidos por medio de la observación directa se utilizará el programa PROMODEL para simular todo el proceso que se realiza dentro de la Central. También se empleará el programa de EXCEL para las debidas entrevistas.

\section{Resultados}

Acorde a la pirámide de los niveles de automatización una empresa de gran magnitud como lo es la Unidad de Negocio Termopichincha Quevedo II CELEC EP debe estar en un nivel 5; con respecto a la entrevista realizada se ha identificado que actualmente la empresa si mantiene un nivel 5 de automatización correspondiente al Nivel de Gestión (ERP) en la mayoría de sus sistemas.

La empresa al mantener un nivel de automatización 5 , está totalmente conformada por sistemas que les permite realizar las funciones de gestión comercial y marketing, planificación estratégica, financiera y administrativa, gestión de recursos humanos, ingeniería del proceso, gestión de sistemas de información y otras. Este nivel emite al Nivel IV información sobre la situación comercial (pedidos y previsiones), información de ingeniería de producto y de proceso, etc. Para poder ajustar la planificación global este nivel recibe del Nivel IV la información anteriormente indicada sobre cumplimiento de programas y costes, etc. Esta área mantiene sistemas automatizados para el control y la supervisión de cada uno de los procesos que se llevan a cabo dentro de la empresa, por medio de un sistema SCADA el cual se encarga de mantener en constante comunicación con los sistemas de campo y los de control.

\section{Área de generación de vapor}


La siguiente grafica representa una rama de control de lazo cerrado, por medio de la caldera de gases alimenta vapor a las purificadoras de combustible donde estas ayuda a reducir las impurezas del combustible bunker (HFO), este sistema a su vez está controlado por un PLC programado para operar mediante el cierre y apertura de cada uno de los sensores y válvulas, lo cual si no hay una entrada de vapor las purificadoras de combustible no funcionan.

Área de generación eléctrica

Esta grafica representa un sistema de control de lazo cerrado, desde los tanques de servicios pasa el fluido a la planta de tratamiento de combustible HTU para elevar la temperatura desde los $80^{\circ} \mathrm{C}$ a los $140^{\circ} \mathrm{C}$; cuando este a esta temperatura ingresa el fluido al motor, el combustible sobrante pasa por una tubería de retorno, lo cual regresa a los tanques de servicios.

\section{Área de enfriamiento}

La siguiente imagen muestra el sistema de enfriamiento del motor el cual viene de los tanques de agua tratada para enfriar cada uno de los componentes del motor, una vez que el agua este a una temperatura de $80^{\circ} \mathrm{C}$ acciona la válvula termostática de agua de alta; dándole apertura para que el agua caliente pase al radiador para ser enfriada en un circuito cerrado, cuando el agua está a una temperatura de $40^{\circ} \mathrm{C}$ la válvula termostática de agua de baja permite el ingreso de agua al motor para otra vez enfriar el sistema, siendo este un sistema de control de lazo cerrado.

Automatizar los procesos de la empresa proporcionara un mayor uso de sistemas o elementos computarizados para controlar maquinarias y/o procesos industriales substituyendo a operadores humanos; la mecanización de los procesos, donde se provee a operadores humanos mecanismos para asistirlos en los esfuerzos físicos del trabajo, y se reduce ampliamente la necesidad sensorial y mental de la persona. Cuando existe automatización en un proceso industrial, el hombre encamina su protagonismo básicamente a la función de supervisión y monitoreo.

Comparación de los procesos manuales y automáticos existentes en la empresa Proceso de recepción de combustible y de agua cruda son procesos manuales porque tienen que ser controlados y visualizados por medio de un operador, en cambio los demás procesos como el de generación de vapor, el sistema de enfriamiento de agua, el sistema de purificación de combustible, generación, distribución y transmisión eléctrica son procesos automatizados porque con la ayuda de un sistema de control reduce los errores humanos aumentando la velocidad de ejecución en los procesos. Por lo general las empresas deberían proveer en la mayor parte de los procesos de sistemas automatizados, esto sería de gran ayuda en la disminución de errores en el proceso, y a su vez optimizaría la seguridad de las instalaciones y la protección de los trabajadores; la Unidad de Negocios Termopichincha Quevedo II CELEC EP al ser una empresa encargada a la generación y distribución de energía eléctrica en la totalidad de sus procesos son automatizados asegurando una mejora en la calidad 
del trabajo del operador y en el desarrollo del proceso, esta dependerá de la eficiencia del sistema implementado.

\section{Procesos que requieren mejoras en automatización.}

El proceso de recepción de agua cruda requiere de una boya, la cual ayuda a que no haya reboses en el tanque de almacenamiento de agua cruda, evitando que un operador este en constante control del sistema de llenado.

La mejora de los procesos, significa optimizar la efectividad y la eficiencia, mejorando también los controles, reforzando los mecanismos internos para responder a las contingencias, al mejorar el proceso de recepción de agua cruda implicará aumento en costos de implementación para toda la empresa pero reducirá tiempos de ejecución en el proceso de llenado de agua cruda, al efectuar un sistema de automatización en el proceso antes mencionado se logrará que el operador solo este supervisando el nivel de llenado, mas no abriendo y cerrando válvulas hasta que se llene el tanque.

\section{Conclusiones}

Se ha logrado identificar el nivel de automatización que mantiene la empresa en la actualidad, y con la ayuda de la entrevista realizada al encargado de la empresa, el Ing. Gabriel Robinson se pudo conocer que la Unidad de negocio Termopichincha Quevedo II CELEC EP se encuentra en un nivel 5 de automatización, el cual corresponde al Nivel de Gestión (ERP) en la mayoría de sus sistemas. La empresa al encontrarse en un nivel ERP, cuenta en la actualidad con la mayoría de sus áreas automatizadas; la cuales son: área de control y supervisión, área de generación de vapor, área de generación eléctrica y área de enfriamiento. Todo esto permite tener control en las maquinarias y procesos efectuados en la planta, evitar errores aumentando la velocidad de ejecución en los procesos, y a la vez prevenir accidentes que afecten la integridad física de los trabajadores. A través de la entrevista y la visita realizada a la empresa se observó que el único proceso efectuado de manera manual es el de recepción de combustible y de agua cruda, en el que se necesita de un operador para ser visualizado y controlado. A diferencia de los demás procesos que si se encuentran automatizados.

Existe un proceso que requiere mejoras en automatización, y corresponde al de recepción de agua cruda. En la actualidad para esta actividad se requiere de un operador que esté en constante control en el llenado para evitar el rebose del tanque.

\section{Referencia}


Blanca, A. (09 de 04 de 2008). Automatización y robótica para la producción. Recuperado el 24 de 11 de 2018, de https://www.gestiopolis.com/automatizacion-robotica-produccion/

Fernández Sánchez, E. (s.f.). Estrategias de Producción. Universidad de Oviedo.

Loayza Pérez, J., \& Silva Meza, V. (10 de 10 de 2013). Diseño y Tecnología.

Recuperado el 24 de 11 de 2018, de file:///C:/Users/equipo/Downloads/642522495-1-PB.pdf

MCR, G. (29 de 07 de 2016). Automatización Industrial. Recuperado el 24 de 11 de 2018, de https://www.mcr.es/ventajas-y-desventajas-de-la-automatizacionindustrial/

Robles, A. C. (2010). MODELO DE GESTIÓN COMERCIAL DE ENERGÍA ELÉCTRICA EN EL ECUADOR. Cuenca.

Sabogal Vanegas, J. T. (15 de 10 de 2012). Importancia de la Automatización. Recuperado el 24 de 11 de 2018, de http://www.redjbm.com/catedra/index.php/tecnologia/68-la-importancia-de-laautomatizacion

School, B. (s.f.). Project Management. Recuperado el 24 de 11 de 2018, de https://www.obs-edu.com/int/blog-project-management/proyectos-deingenieria/que-caracteristicas-tiene-un-proceso-industrial

Siemens, I. (s.f.). Tecnología e incremento de la competitividad industrial. SIEMENS, 2-3.

Taipe Flores, F. P. (2011). El sector eléctrico ecuatoriano en los últimos 20 años. Quito: Pontificia Universidad Católica del Ecuador.

teacherke. (09 de 2010). Introducción a la Automatización. Recuperado el 24 de 11 de 2018, de https://teacherke.files.wordpress.com/2010/09/introduccion-a-laautomatizacion.pdf 
Centro Sur. Social Science Journal. eISSN: 2600-5743. Julio - Diciembre Vol. 4 No. 2 - 2020 http://centrosureditorial.com/index.php/revista 